\title{
The electron affinity of tellurium
}

\author{
Gunnar Haeffler, Andreas E. Klinkmüller, Jonas Rangell, Uldis Berzinsh and Dag Hanstorp \\ Dept. of Physics, Göteborg University and Chalmers University of Technology, S-41296 Göteborg, Sweden
}

June 22, 2021

\begin{abstract}
The electron affinity of tellurium has been determined to $1.970876(7) \mathrm{eV}$. The threshold for photodetachment of $\mathrm{Te}^{-}\left({ }^{2} P_{3 / 2}\right)$ forming neutral $\mathrm{Te}$ in the ground state was investigated by measuring the total photodetachment cross section using a collinear laser-ion beam geometry. The electron affinity was obtained from a fit to the Wigner law in the threshold region.
\end{abstract}

PACS: $35.10 . \mathrm{H}, 32.80 . \mathrm{F}$

\section{Introduction}

Negative ions are fragile quantum systems, which differ considerably from neutral atoms and positive ions in numerous important aspects [1], 2]. Most prominently the binding energy of the outermost electron is substantially lower than in iso-electronic atoms due to the more efficient screening of the nuclear charge by the other electrons [3]. This increases the significance of correlation effects for the outermost electrons and makes negative ions a crit-

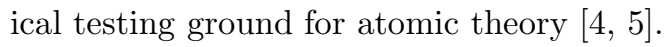

The short range potential of negative ions typically only sustains one bound state 3]. In the rare cases with more than one bound state they are of the same parity as the ground state and consequently inaccessible to one-photon electric dipole transitions. Hence, the electron affinity (EA) is one of very few properties of a negative ion that can be determined with high accuracy. The potentially most accurate method to determine EA is the so called laser photodetachment threshold (LPT) method [6] in a collinear geometry. In more recent years we have applied this method to Iodine [7] to determine the EA and to Chlorine 8 where we measured both the EA and the isotope shift between ${ }^{35} \mathrm{Cl}^{-}$and ${ }^{37} \mathrm{Cl}^{-}$.

In this paper we investigate the EA of Tellurium. If comparing with other elements with a relatively high electron affinity this quantity is determined with a relatively large uncertainty [3, 9, 10]. The aim of this work is to achieve an improvement of this value by using the LPT method in a collinear geometry.

\section{Experiment}

\section{$2.1 \quad$ Setup}

A collinear laser-ion beam setup, schematically shown in Fig. 11, was used. The setup is described in more detail elsewhere [11, 12]. ${ }^{130} \mathrm{Te}^{-}$ions were produced in a Cs-sputter ion source and accelerated to an energy of approximately $4 \mathrm{keV}$. The ions were subsequently mass-analyzed before entering the interaction-detection chamber, which was kept under a pressure of approximately $7 \times 10^{-9}$ mbar $\left(7 \times 10^{-7} \mathrm{~Pa}\right)$. An electrostatic quadrupole deflector guided the ion beam into the chamber thereby allowing a collinear laser-ion beam interaction. The interaction region was defined by two apertures with a diameter of $3 \mathrm{~mm}$ placed $0.5 \mathrm{~m}$ apart. A second electrostatic quadrupole deflector separated ions from atoms after the interaction region and the ion current was measured in a Faraday cup. The ion current was typically $1 \mathrm{nA}$. Atoms created in the interaction region continued through the sec- 


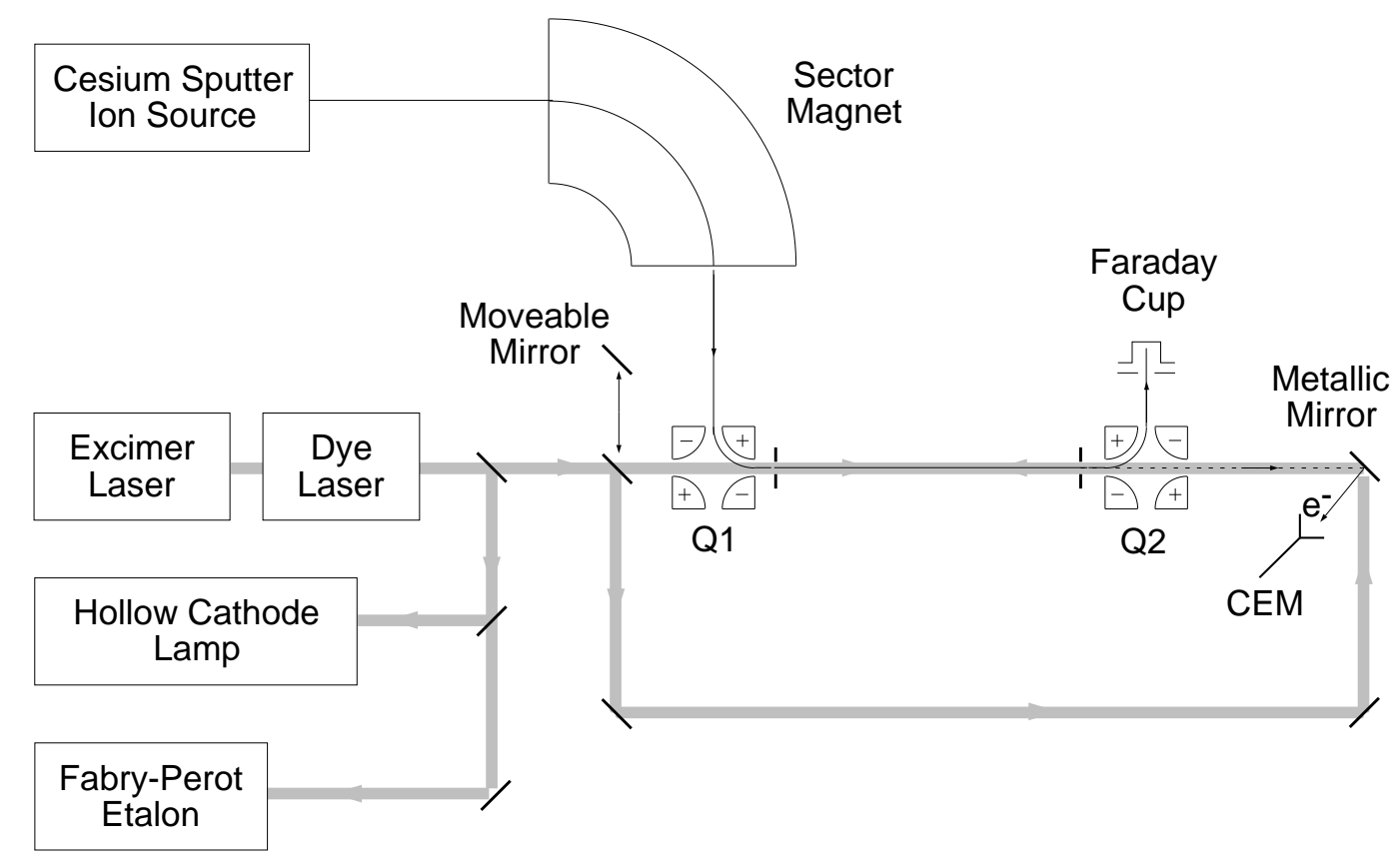

Figure 1: Experimental setup: Schematic diagram of the collinear laser and ion beam setup. The movable mirror is used to reverse the direction of the laser beam during a scan.

ond electrostatic quadrupole deflector and were detected using a neutral-particle detector capable of detecting single atoms. Only atoms arriving during a $6 \mu$ s gate after the laser pulse were detected. This time-gated detection scheme was used to suppress the background mainly caused by collisional detachment. The detector as well as the detection scheme have been described in detail elsewhere [13].

A tunable dye laser pumped by an excimer laser and operated with Rhodamine B was used to generate the laser light. The pulse duration was approximately $15 \mathrm{~ns}$ and the pulse energy used in the experiment was typically $10 \mu \mathrm{J}$. The frequency of the light was determined by combining Fabry-Perot fringes and optogalvanic spectroscopy using hollow cathode lamps.

A new computer program for data acquisition and laser control by a personal computer (PC) was developed. The dye laser was controlled via a general purpose interface bus (GPIB). The program allowed scanning of the laser frequency with different step size and different number of laser shots per step within the same scan. The pulses from a channel electron multiplier (CEM) were counted by a gated photon counter connected to a $\mathrm{PC}$ via a GPIB. The signals used for calibration were measured by means of boxcar integrators and transfered to the computer via $\mathrm{A} / \mathrm{D}$ converters.

\subsection{Procedure}

The electron affinity of Te corresponds to the photon energy needed to reach the first threshold for photodetachment of the $\mathrm{Te}^{-}$ground state, a process that can be denoted as

$$
T e^{-}\left(5 p^{5}{ }^{2} P_{3 / 2}\right)+\hbar \omega \rightarrow T e\left(5 p^{4}{ }^{3} P_{2}\right)+e^{-} .
$$

The angular momentum of the outgoing electron in the vicinity of the threshold is predominantly $l=0$. As seen in Fig. 2, there will be additional contribution to the residual atom yield due to the processes

$$
\begin{aligned}
& T e^{-}\left(5 p^{5}{ }^{2} P_{1 / 2}\right)+\hbar \omega \rightarrow T e\left(5 p^{4} P_{2}\right)+e^{-},(2) \\
& T e^{-}\left(5 p^{5} P_{1 / 2}\right)+\hbar \omega \rightarrow T e\left(5 p^{4} P_{1}\right)+e^{-}, \\
& T e^{-}\left(5 p^{5} P_{1 / 2}\right)+\hbar \omega \rightarrow T e\left(5 p^{4} P_{0}\right)+e^{-} .
\end{aligned}
$$

The contribution of these processes to the signal, however, varies slowly with the photon energy since 


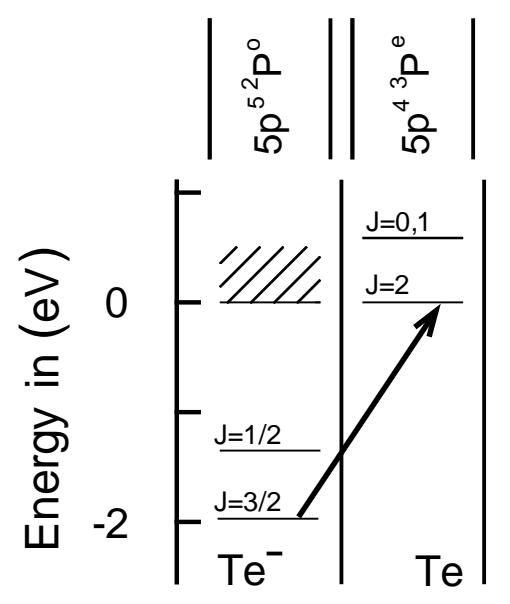

Figure 2: Excitation scheme: Selected states of $\mathrm{Te} / \mathrm{Te}^{-}$. The arrow indicates the transition induced in this experiment.

they are far above their thresholds. Furthermore, these signals are relatively small since the sputter ion source predominantly produces ground state negative ions. The approach in this work was therefore to determine the electron affinity by performing a LPT measurement around the threshold for the process described by (11), using a collinear laserion beam geometry.

The total photodetachment cross section was obtained by measuring the neutral atom yield as a function of the laser frequency. To establish the frequency scale, the optogalvanic signal from a hollow cathode lamp and the fringes from a Fabry-Perot etalon were recorded simultaneously with the neutral atom signal as the laser frequency was tuned. The Fabry-Perot fringes served as frequency markers whereas atomic transitions in $\mathrm{Ne}$ 14, 15 or $\mathrm{Ar}$ [16. from two different hollow cathode lamps provided an absolute calibration of the scale. Within one frequency scan only one hollow cathode lamp was used, but by performing a number of scans with each lamp a control of the frequency calibration was obtained. The direction of the laser beam was reversed within each scan to obtain the photodetachment cross section both for the case of parallel ion and laser beams, where the photon energy seen by the ions is red-shifted due to the Doppler shift, as well as in the case of anti-parallel beams, where a blue-shift is obtained. Each scan covered a fre-

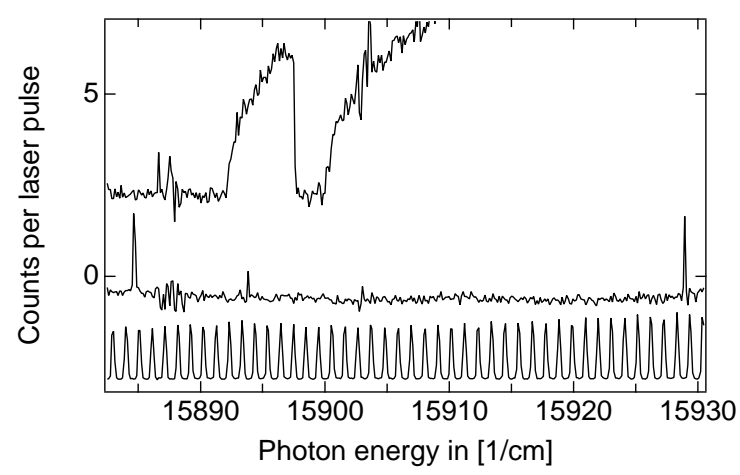

Figure 3: Scan overview: The three curves show the photodetachment signal, the reference lines (Ne) and the Fabry-Perot fringes. In the photodetachment signal, the left threshold is obtained with antiparallel laser and ion beams and the right threshold with parallel beams. The background is partly due to photodetachment of the $\mathrm{Te}^{-}\left(5 p^{5}{ }^{2} \mathrm{P}_{1 / 2}\right)$ state. The signal saturates at about 7 counts per laser pulse. In the vicinity of the calibration lines and the photodetachment thresholds the step-length was $1 \mathrm{pm}$ and 100 shots per point were taken. Elsewhere the step-length was $5 \mathrm{pm}$ and 10 shots per point were taken. The vertical scale (Counts per laser pulse) is only valid for the photodetachment signal.

quency range wide enough to record one identified transition in $\mathrm{Ar}$ or $\mathrm{Ne}$ in the optogalvanic spectrum below and one above the two Doppler shifted thresholds.

A larger frequency step size and a smaller number of laser pulses were used in the frequency ranges between the calibration lines and the thresholds where the only relevant information was the number of Fabry-Perot fringes to keep track of the relative frequency scale. In this manner the scanning time was reduced and hence any long time drifts of the system were minimized.

\section{Results}

The result of a typical measurement of the neutral atom signal, fringes from the Fabry-Perot etalon and the signal from optogalvanic spectroscopy, using a hollow cathode lamp is shown in Fig. 3. 
Two thresholds, both representing photodetachment from the $\mathrm{Te}^{-}\left({ }^{2} P_{3 / 2}\right)$ ground state through the process described in (11), are seen in the spectrum. At lower photon energies the laser and ion beams propagate anti-parallel. The direction of the laser beam was reversed at a photon energy of about $15882 \mathrm{~cm}^{-1}$ hence giving a second measurement of the now blue shifted threshold. By the use of Ar transitions for the frequency calibration a typical scanning range was $629.882 \mathrm{~nm}$ to $628.022 \mathrm{~nm}$ $\left(15876 \mathrm{~cm}^{-1}\right.$ to $\left.15923 \mathrm{~cm}^{-1}\right)$ and in the case of Ne transitions the scanning range was $629.564 \mathrm{~nm}$ to $627.746 \mathrm{~nm}\left(15884 \mathrm{~cm}^{-1}\right.$ to $15930 \mathrm{~cm}^{-1}$ ) (the used reference lines are tabulated in Tab. 21). In regions close to optogalvanic signal peaks and photodetachment thresholds the laser wavelength step was $1 \mathrm{pm}$ and in between, where it only was important to keep track of the number of Fabry-Perot fringes, the step size was increased to $5 \mathrm{pm}$. In the vicinity of the photodetachment threshold 100 laser pulses were used for each frequency step whereas this number was decreased to 10 in other regions.

To obtain the photodetachment threshold energy $E_{0}$ we fitted the Wigner law [17] for $s$-wave detachment,

$$
\sigma(E)= \begin{cases}a+b \sqrt{E-E_{t h}}, & E>E_{t h}, \\ a, & E<E_{t h}\end{cases}
$$

to our data by adjusting the parameters: $a$ for the non-resonant background, $b$ for the cross section amplitude and $E_{t h}$ for the threshold energy. The value of the threshold energy parameter is either blue shifted $E_{0}^{b}$, for parallel laser and ion beams, or red shifted $E_{0}^{r}$, for anti-parallel laser and ion beams (Fig. 3 and 4). To attain a threshold energy, $E_{0}$, corrected for the Doppler shift to all orders the geometric mean of the red and blue shifted threshold energy has to be taken:

$$
E_{0}=\sqrt{E_{0}^{b} E_{0}^{r}}
$$

The final value, $\overline{E_{0}}=15896.18(5) \mathrm{cm}^{-1}$ is a weighted average of eight measurements with frequency calibration obtained from Ar transitions and ten measurements using Ne transitions. Average values have also been calculated individually for the sets of $E_{0}$ values obtained by calibrating to $\mathrm{Ne}$ and Ar transitions respectively. These values are presented in Tab. 1.

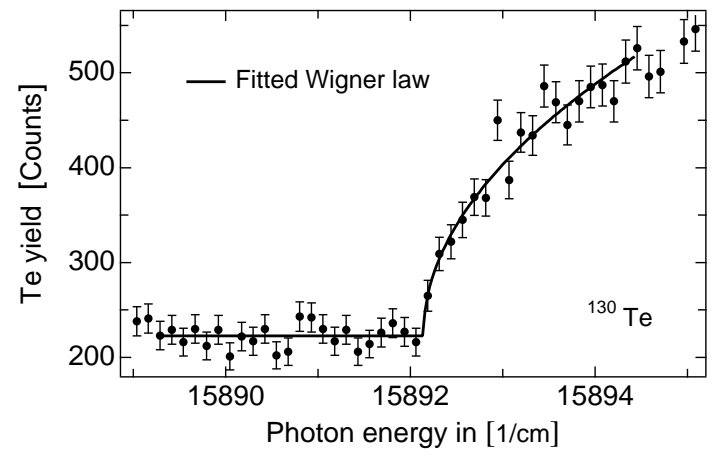

Figure 4: $\mathrm{Te}\left(5 p^{4}\right)+\epsilon s$ threshold: Measurement of the relative total photodetachment cross section around the $\mathrm{Te}\left(5 p^{4}\right)+\epsilon s$ threshold with anti-parallel laser and ion beams. The solid line is a fit of the Wigner law (3) to the experimental data. The error bars represent the shot noise. Each data point was acquired during 100 laser pulses. The background is mainly due to photodetachment of $\mathrm{Te}^{-}\left({ }^{2} P_{1 / 2}\right)$ through the processes described in (2).

There are two major contributions to the uncertainty of $E_{0}$. There is a statistical error of $0.01 \mathrm{~cm}^{-1}$ corresponding to the spread of fitted threshold values. Second, there is an uncertainty related to the laser intensity profile. We have estimated this uncertainty by analyzing Fabry-Perot fringes and the atomic reference lines to be less than $0.04 \mathrm{~cm}^{-1}$, which is one fifth of the laser frequency bandwidth.

To converted our value of $\overline{E_{0}}$ from $\mathrm{cm}^{-1}$ to $\mathrm{eV}$ we used the recommended factor of $(1 / 8065.5410)$ $\left[\mathrm{eV} /\left(\mathrm{cm}^{-1}\right)\right]$ 18] yielding the value $1.970876(7) \mathrm{eV}$ for the EA.

\section{Discussion}

We have obtained an electron affinity of $1.970876(7) \mathrm{eV}$. The uncertainty is of the same order of magnitude as the laser bandwidth. We have thus shown that the background contribution from photodetachment of $\mathrm{Te}^{-}\left({ }^{2} P_{1 / 2}\right)$ is not critical for the accuracy achievable with our laser system. An important part of this experiment was the use of a computer program that can vary both the wavelength-step and the acquisition times for 


\begin{tabular}{lcl}
\hline \hline Ref. line & \# of spectra & EA in $\mathrm{eV}$ \\
\hline Neon & 10 & $1.9708780(14)$ \\
$\mathrm{Ar}$ & 8 & $1.9708746(22)$ \\
All & 18 & $1.9708764(13)$ \\
Final (with systematic errors) & $1.970876(7)$ \\
\hline \hline
\end{tabular}

Table 1: EA of Te: The two first lines shows the values obtained using transitions in Neon and Argon for the wavelength calibration, and the third line shows their weighted average. The uncertainty given is then only the statistical (one sigma). In our final value the estimated systematic uncertainty is included as described in the text.

\begin{tabular}{|c|c|}
\hline$\overline{\overline{\text { Element }}}$ & "Line $\left(\mathrm{cm}^{-1}\right)$ \\
\hline $\mathrm{Ne} \mid 15,14$ & $15929.216(4)$ \\
\hline $\mathrm{Ne} 15,14$ & $15884.396(6)$ \\
\hline Ar $\sqrt{16}$ & $15922.598(5)$ \\
\hline Ar $\sqrt{16}$ & $15876.508(5)$ \\
\hline
\end{tabular}

Table 2: Calibration lines: Transition energy of the calibration lines used in this experiment. The Ne transitions are $\left({ }^{2} P_{3 / 2}\right) 4 d[1 / 2] J=0 \rightarrow$ $\left({ }^{2} P_{1 / 2}\right) 3 p[1 / 2] J=1$ and $\left({ }^{2} P_{1 / 2}\right) 5 s[1 / 2] J=1 \rightarrow$ $\left({ }^{2} P_{1 / 2}\right) 3 p[3 / 2] J=1$, were all involved lines are $2 p^{5} \mathrm{nl}$. We calculated the transition energies from the tabulated interferometrically 14 or by Fourier transform spectroscopy 15] determined level energies. The Ar transition energies are calculated from interferometrically determined levels, as presented in Tab $\mathrm{V}$ of [16] .

each wavelength. This has proven to be most valuable in order to improve the statistics and simultaneously decrease the total acquisition time, hence diminishing the probability of long term drifts. The value we have achieved is of comparable accuracy as the best EA determinations [2, 3], and our two independent calibrations add reliability to this measurement.

This new value of the electron affinity of Tellurium is 45 times more accurate than the value of Slater [9] and fall well within their error-bars. The more recent value of Thøgersen [10] agrees with ours within two error bars.

\section{Acknowledgments}

Financial support for this research project has been obtained from the Swedish Natural Science Council (NFR). Personal support was received from Chalmers University of Technology for Uldis Berzinsh. R. L. Kurucz at Harvard-Smithsonian Center for Astrophysics is gratefully acknowledge for supply us with their Atomic Line List.

\section{References}

[1] S. J. Buckman and C. W. Clark, Rev. Mod. Phys. 66, 539 (1994).

[2] C. Blondel, Physica Scripta T58, 31 (1995).

[3] H. Hotop and W. C. Lineberger, J. Phys. Chem. Ref. Data 14, 731 (1985).

[4] S. Salomonson, H. Warston, and I. Lindgren, Phys. Rev. Lett. 76, 3092 (1996).

[5] C. F. Fischer and T. Brage, Can. J. Phys. 71, 1283 (1992).

[6] D. M. Neumark, K. R. Lykke, T. Andersen, and W. C. Lineberger, Phys. Rev. A 32, 1890 (1985).

[7] D. Hanstorp and M. Gustafsson, J. of Phys. B: At., Mol. Opt. 25, 1773 (1992).

[8] U. Berzinsh et al., Phys. Rev. A 51, 231 (1995).

[9] J. Slater and W. C. Lineberger, Phys. Rev. A 15, 2277 (1977).

[10] J. Thøgersen et al., Phys. Rev. A 53, 3023 (1996).

[11] U. Ljungblad, A. Klinkmüller, and D. Hanstorp, A new apparatus for studies of negative ions, in Fifth european workshop on the production and application of light negative ions, edited by M. Hopkins and S. Fahy, pages 35-40, Glasnevin, Dublin 9, Ireland, 1994, Dublin City University.

[12] D. Hanstorp, Nucl. Instrum. Methods Phys. Research 100, 165 (1995). 
[13] D. Hanstorp, Meas. Sci. Technol. 3, 523 (1992).

[14] V. Kaufman and L. Minnhagen, J. Opt. Soc. Am. 62, 92 (1972).

[15] E. S. Chang and W. G. Schoenfeld, Physica Scripta 49, 26 (1994).

[16] L. Minnhagen, J. Opt. Soc. Am. 63, 1185 (1973).

[17] E. P. Wigner, Phys. Rev. 73, 1002 (1948).

[18] E. R. Cohen and B. N. Taylor, J. Phys. Chem. Ref. Data 17, 1795 (1988). 